\title{
Localized holographic recording in doubly doped lithium niobate
}

Christophe Moser, Benjamin Schupp, Irena Maravic, Demetri Psaltis

Christophe Moser, Benjamin Schupp, Irena Maravic, Demetri Psaltis, "Localized holographic recording in doubly doped lithium niobate," Proc. SPIE 4089, Optics in Computing 2000, (24 May 2000); doi: 10.1117/12.386813

Event: 2000 International Topical Meeting on Optics in Computing (OC2000), 2000, Quebec City, Canada 


\title{
Localized Holographic Recording in doubly doped Lithium Niobate
}

\author{
Christophe Moser*, Benjamin Schupp, Irena Maravic,Demetri Psaltis \\ California Institute of Technology \\ Department of Electrical Engineering,Pasadena, CA 91125
}

\begin{abstract}
In holographic data storage, pages of information are overlapped in the volume of the recording medium. Due to destructive read-out of holograms in photorefractive crystals such as $\mathrm{LiNbO}_{3}: \mathrm{Fe}$, holograms are recorded with an exposure schedule in order to equalize diffraction efficiency. This leads to a final diffraction efficiency proportional to $1 / \mathrm{M}^{2}$, where $\mathrm{M}$ is the number of exposures. Coherent erasure of a particular page also erases all the other pages stored in the same volume. We believe to have found a technique that does not require an exposure schedule and that can record $\mathrm{M}$ holograms with diffraction efficiency following a $1 / \mathrm{M}$ dependance. Our technique is based on non-destructive read-out in doubly-doped $\mathrm{LiNbO}_{3}[1]$. The technique is based on the recording of localized holograms in thin layers across the volume of the crystal.
\end{abstract}

Keywords: doubly-doped $\mathrm{LiNbO}_{3}$, localized holograms, data storage

\section{INTRODUCTION}

In holographic data storage [1], pages of information are overlapped in the volume of the recording medium. Coherent erasure of a particular page also erases all the other pages stored in the same volume, due to destructive read-out of holograms in photorefractive crystals such as $\mathrm{LiNbO}_{3}: \mathrm{Fe}$. Recently, non-destructive read-out has been demonstrated in doubly-doped $\mathrm{LiNbO}_{3}$ [2]. When volume holograms are multiplexed in such crystals [3], the erasure of a single page of information requires the sensitizing light to be present, causing the erasure of all the other pages of information stored in the same volume. Here, we propose to holographically record localized holograms in doubly doped $\mathrm{LiNbO}_{3}[4]$ The method can be applied to any other material that produces non-volatile read-out $[5,6,7]$. The method can be considered the holographic analog of the three-dimensional optical two-photon memories architecture [8,9]. In the first portion of the paper, we experimentally demonstrate the recording of 100 localized holograms in doubly-doped $\mathrm{LiNbO}_{3}$. We quantify the quality of the reconstruction by measuring the signal to noise ratio (SNR) of the recorded holograms. These localized holograms can be erased and refreshed selectively by illuminating the hologram with the sensitizing and reference beam. We present theoretical results on the diffraction efficiency obtainable and the total recording time for $\mathrm{M}$ holograms and compare those results with the conventional distributed volume recording. We show that, when the modulation depth of the holograms is maximized, the final diffraction efficiency after $M$ localized recordings varies as $1 / M$. This behavior represents a large improvement compared to the $1 / \mathrm{M}^{2}$ dependance obtained in distributed volume recording. An experiment is presented that confirms those predictions.

\section{LOCALIZED RECORDING}

\subsection{SNR of 100 localized holograms}

The set-up for SNR measurement is shown in fig 1 . The reference beam is focused by a cylindrical lens of focal length $4 \mathrm{~cm}$. The blue beam from a laser diode (Nichia, $401 \mathrm{~nm}$ ) is co-propagating with the green $(532 \mathrm{~nm})$ reference beam. A Spatial light Modulator (SLM) of $640 \times 480$ pixels is used to display random binary patterns. The 4-f imaging system is not pixel matched, so that $4 \times 4$ SLM pixels are used to represent 1 data pixel. The imaging system consists of an achromat lens with N.A= 0.4 placed in front of the SLM and an objective Nikon lens with N.A=1.4. The imaging system's magnification of $\sim 0.3$ allows to image the entire SLM surface onto the camera. A removable mirror can be placed after the second imaging lens to measure the diffracted power while leaving the camera in place. The holograms are recorded in the Fresnel region. The focal plane of the first lens is located $3 \mathrm{~mm}$ before the crystal's surface. An iris is placed at the focal plane of the first imaging lens to filter out the high orders generated by the SLM. The signal beam propagates through the crystal close to one of its edges. This is to ensure that the violet light sensitizes efficiently the area to be recorded from the side as shown in the inset of fig 1 . The holograms are recorded from position 1 (exit crystal facet) to M (entrance crystal facet).

\footnotetext{
* Correspondance: Email: moser@sunoptics.caltech.edu;Telephone: 626395 3159; Fax: 6265688437
} 


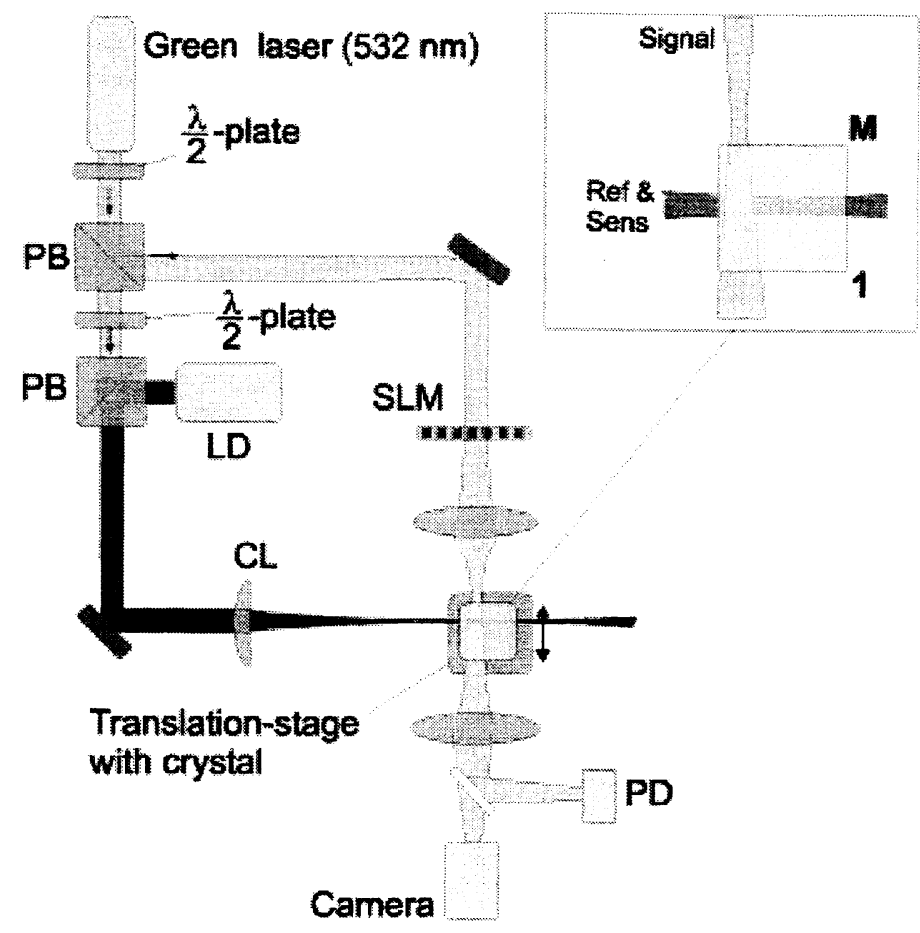

Fig 1: Recording set-up for the SNR measurement

This way, the reference beam interferes with the signal beam before it propagates through the previously recorded holograms, thus reducing distortion of the signal. At the overlap between the focused reference and the signal beam, the signal diameter is $\sim 1.5 \mathrm{~mm}$. The experiment is performed with a congruent 90 degree-cut $\mathrm{LiNbO}_{3}$ crystal doped with 0.15 wt. $\% \mathrm{Fe}_{2} \mathrm{O}_{3}$ and $0.015 \mathrm{wt} . \% \mathrm{MnO}$. The crystal dimensions are $1 \times 1 \times 1 \mathrm{~cm}^{3}$. The recording curve using two extended plane waves is shown in fig.2.

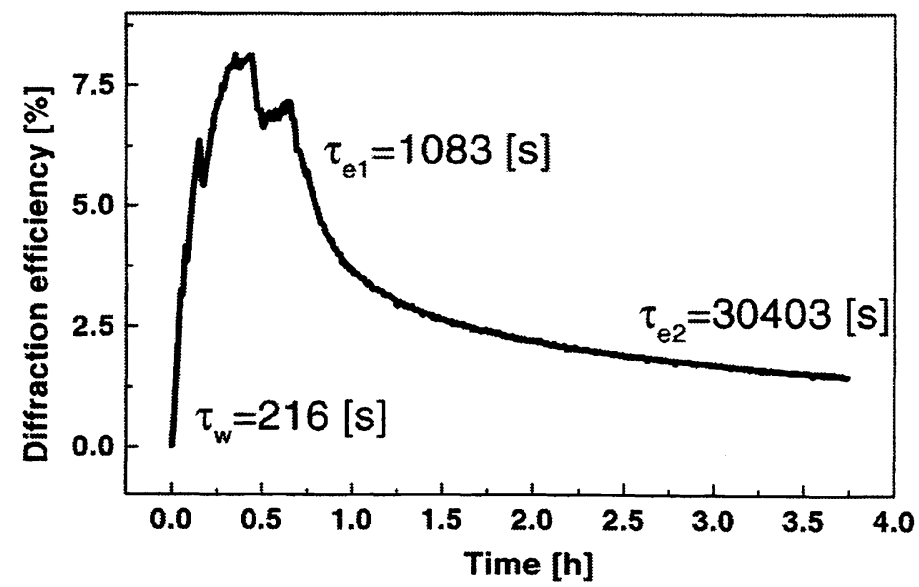

Figure 2: Recording and read-out curve for a plane wave hologram in a 90 degree-cut $\mathrm{LiNbO}^{3}$ crystal doped with $0.15 \mathrm{wt} . \% \mathrm{Fe}_{2} \mathrm{O}_{3}$ and $0.015 \mathrm{wt} . \% \mathrm{MnO}$. The recording beams are from a solid state green laser (wavelength $532 \mathrm{~nm}$, ordinary polarization, $1 / \mathrm{e}^{2}$ beam diameter $2.0 \mathrm{~mm}$ and intensity $160 \mathrm{~mW} / \mathrm{cm}^{2}$ ). The sensitizing light is from a laser diode from Nichia, wavelength $401 \mathrm{~nm}$, linearly polarized, intensity $25 \mathrm{~mW} / \mathrm{c} \mathrm{m}^{2}$. 
The erasing curve is fitted by a bi-exponential function giving two time constants, $\tau_{\mathrm{e} 1}=1,083[\mathrm{~s}]$ and $\tau_{\mathrm{e} 2}=30,403$ [s] corresponding respectively to the fast erasure of the grating in $\mathrm{Fe}$ and a slower erasure of the grating in $\mathrm{Mn}$. The crystal was oxidized for 6 hours at $900^{\circ} \mathrm{C}$ in $\mathrm{O}_{2}$ atmosphere. The oxidation state could not be further reduced by longer oxidation. The slow erasure time constant $\tau_{\mathrm{e} 2}$ is about 15 times shorter than that obtained in a similar crystal with half the concentration of $\mathrm{Fe}_{2} \mathrm{O}_{3}$. However. since the recording is performed with equal power in reference and signal beam. the signal intensity is much less than the reference intensity. With the intensities used in the experiment. we obtain a ratio $\tau_{\mathrm{c} 2} / \tau_{\mathrm{w}}=140$, large enough to record hundreds of localized holograms. This ratio is measured by recording the localized hologram to saturation and by letting the signal beam erase the hologram.

In the experiment of fig. 1 , the spot size $2 \mathrm{~W}_{\mathrm{o}}$ has lateral extension $25 \mu \mathrm{m}$ ( intensity $1 / \mathrm{e}^{2}$ value). The waist $\mathrm{W}(\mathrm{z})$ in the crystal increases with distance $z$ as:

$$
W(z)=W_{o} \sqrt{1+\left(\frac{z}{z_{o}}\right)^{2}}, \quad z_{o}=\frac{\pi W_{o}^{2} n}{\lambda_{o}}
$$

where $z_{0}$ is the depth of focus. For a propagation distance of $z=3 \mathrm{~mm}$ inside the crystal, the waist increases to $45 \mu \mathrm{m}$.

The signal, reference and violet beam intensities are $20 \mathrm{~mW} / \mathrm{cm}^{2}, 200 \mathrm{~mW} / \mathrm{cm}^{2}, 40 \mathrm{~mW} / \mathrm{cm}^{2}$ respectively. This choice of intensities results in equal power for reference and signal beam, which yields fast recording as will be shown later. In this experiment, we are interesting in obtaining just enough diffraction efficiency to compute the SNR. A diffraction efficiency of $5 \cdot 10^{-5}$ gives a good image on a video camera sampling at $30 \mathrm{~Hz}$. During recording of subsequent holograms, the previous holograms are partially erased by the green signal beam, but not by the reference beam unlike distributed recording. The spacing between each localized holograms is chosen to be twice the lateral spot size i.e $50 \mu \mathrm{m}$ to avoid erasure of the previous hologram while sensitizing the next hologram. Each hologram is sensitized for $5 \mathrm{~min}$. and recorded for $3 \mathrm{~min}$ and recorded with a different random binary bit pattern. $4 \times 4$ camera pixels represent 1 bit of information. Fig. 3 shows the diffraction efficiency versus position for the 100 holograms. Each peak corresponds to the diffraction efficiency of a hologram stored at a different spatial location as the crystal is translated in the direction perpendicular to the reference beam. The recording did not follow an exposure schedule.

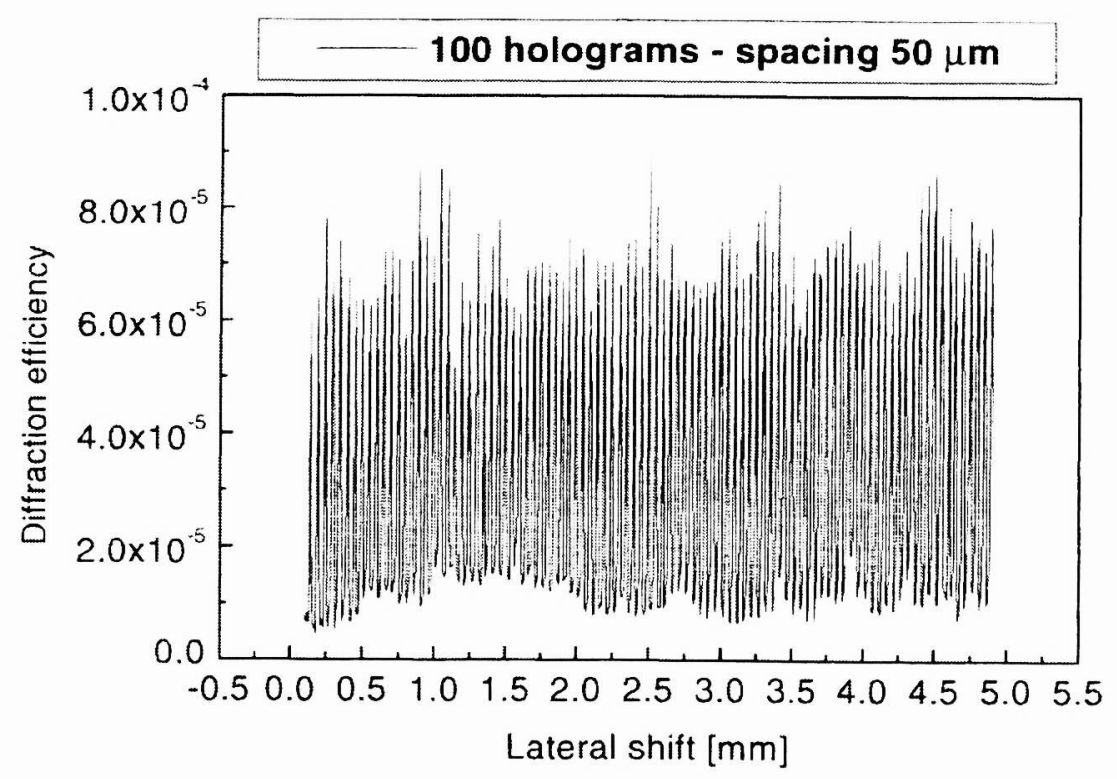

Figure 3: Spatial multiplexing of 100 localized holograms 

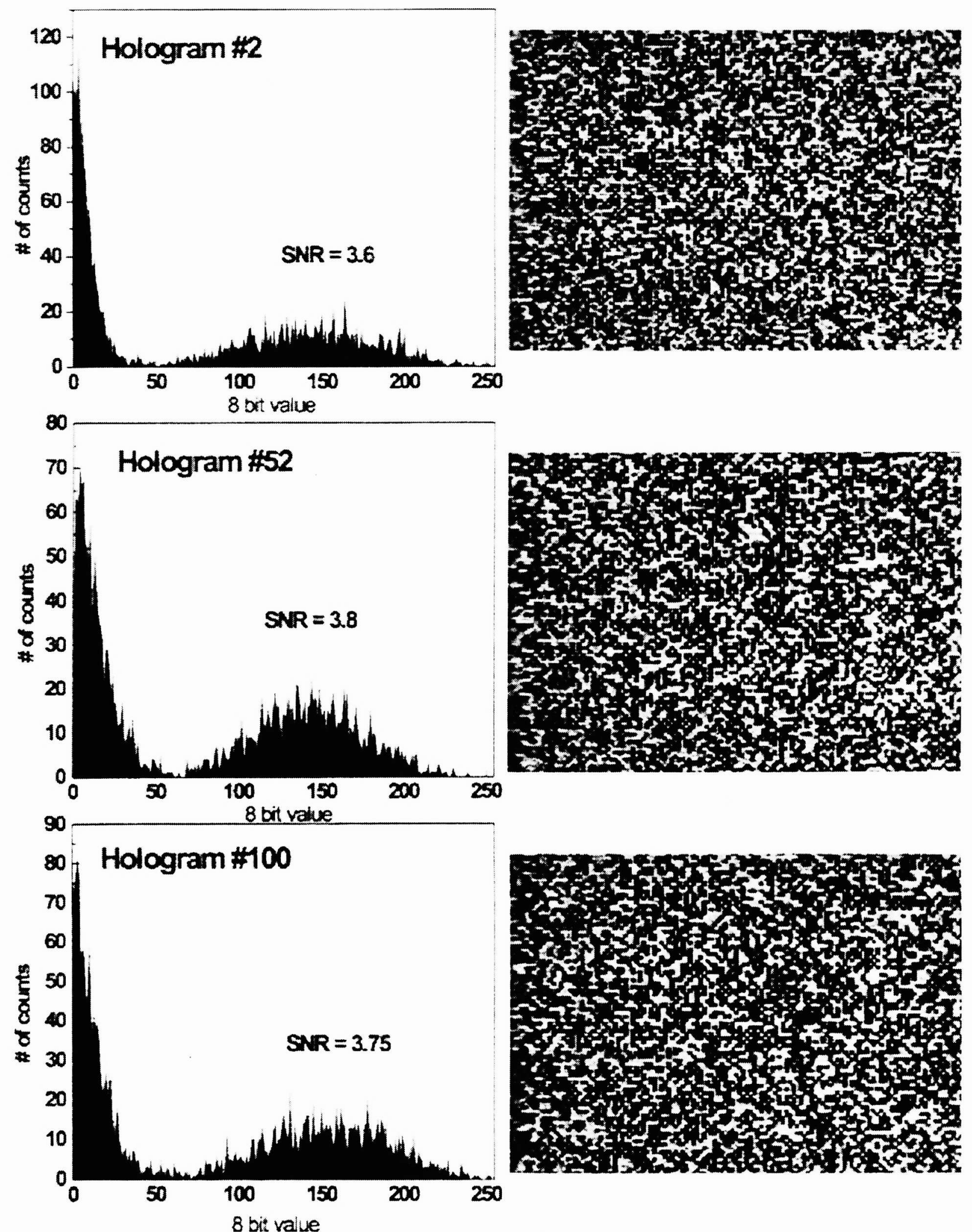

Figure 4: 3 sample reconstructions and SNR values from the 100 holograms experiment 
To measure the SNR, we use 5 windows of $30 \times 30$ superpixels ( 1 superpixel is composed of $4 \times 4$ camera pixels) located on the 4 corners and at the center of the image. For each superpixel, the edges are left out, so effectively $3 \times 3$ pixels form a superpixel. SNR is computed using the formula:

$$
S N R=\frac{\mu_{1}-\mu_{o}}{\sqrt{\sigma_{1}^{2}+\sigma_{2}^{2}}}
$$

Where $\mu_{\mathrm{i}}, \sigma_{\mathrm{i}}, \mathrm{i}=1,0$ are the mean and variance respectively of the fitted Gaussian distribution of the 1's and zero's.

3 sample holograms respectively located at the front, middle, rear of the crystal are shown in fig 4 . We find that the average SNR values measured is 3.7 and constant throughout the crystal. This value is less than the SNR value of 4.2 measured with a focused reference of $100 \mu \mathrm{m}$. Because the SNR is constant for holograms along the crystal, we attribute this loss to the longer exposure of the crystal to the signal beam. The remnant electrons in Fe act as absorbtion centers for the green (532nm) light and produces fanning. The direct imaging of the SLM through the crystal after the storage of 100 hologram shows a degradation of the SNR by 1 compared to the SNR measured before storage. The signal beam intensity varies along the crystal. The intensity is higher at the entrance face of the crystal than the exit face by a factor 15 . Before the memory is written, the first hologram recorded has SNR 4.7 as illustrated in fig. 5.

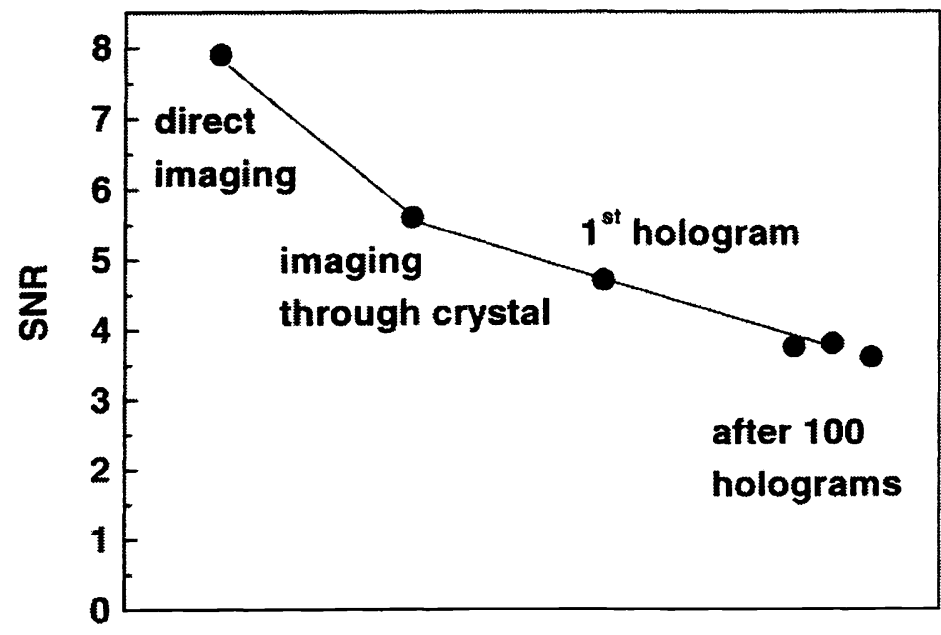

Figure 5: SNR comparison: (a) direct imaging (b) imaging through crystal(c) first hologram (d) after 100 holograms 


\subsection{Diffraction efficiency of localized holograms}

The recording dynamics of localized holograms depends on the relative power distribution between the reference and the signal beam since the recording beam areas are very different. The recording slope $\mathbf{S}$ in photorefractives is given by:

$$
S=\frac{A_{o}}{\tau_{w}} \propto \sqrt{P_{r} P_{S}}
$$

where $P_{r}$ and $P_{s}$ are the power in the reference and signal beam respectively. Eq. 1 is subject to $P_{r}+P_{s}=P_{\text {total }}=$ constant. Thus the recording slope is maximized for equalized power $P_{r}=P_{s}$. In this case the recording slope is optimized but the saturation diffraction efficiency is low because of the low resulting modulation depth. The saturation diffraction efficiency $A_{o}$ is proportional to the modulation depth $\mathrm{m}$ :

$$
A_{o} \propto m=\frac{\sqrt{I_{r} I_{s}}}{I_{r}+I_{s}}
$$

This quantity is maximized for equal intensity $I_{r}=I_{s}$ independant of the total power.

The following experiment illustrates this dynamic. We used a 90 degree--cut $\mathrm{LiNbO}_{3}$ crystal doped with 0.01 wt. $\% \mathrm{Fe}_{2} \mathrm{O}_{3}$. We used a Coherent solid state laser, wavelength $532 \mathrm{~nm}$, linearly polarized, for the recording beams. A cylindrical lens focuses the reference beam ( $6 \mathrm{~mm}$ diameter) in the crystal. The reference beam interferes with a plane wave signal beam ( 6 $\mathrm{mm}$ diameter) in the similar set-up used for the SNR measurement (fig.1). The total power is distributed into the reference and signal beam using a half wave-plate and a polarizing beam splitter. In the first experiment, the reference and signal beam have equal power.

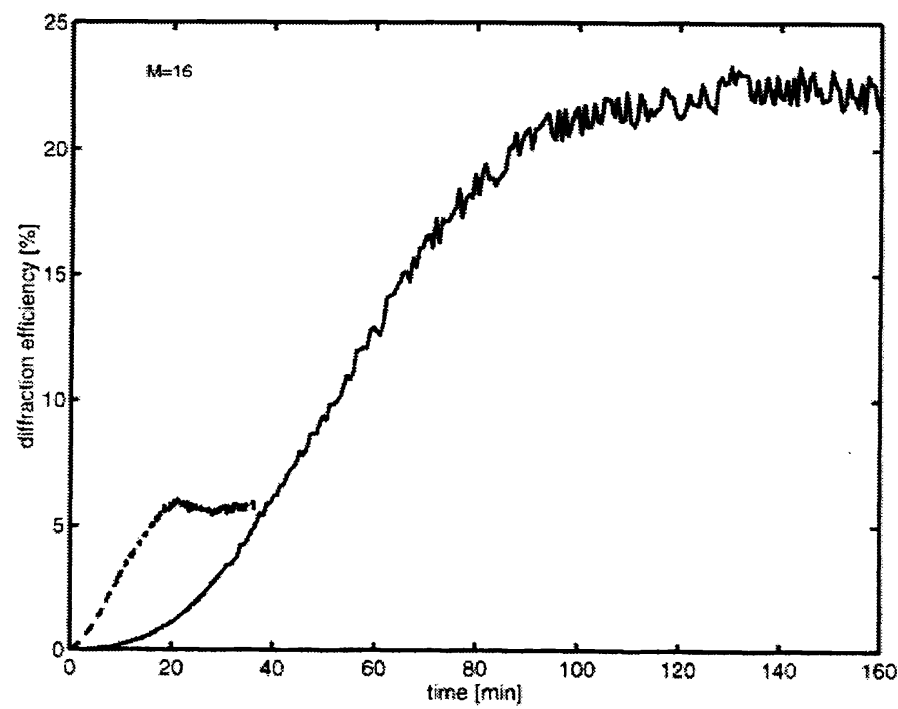

Figure 6: Recording curves of a localized hologram. The plain curve is recorded with equal intensity in the reference and signal beam. The dashed curve is recorded with equal power.

In the second experiment, intensity between the reference and signal beam is equalized. The result is shown in fig. 6 for the case of a reference beam focused to a lateral extension equal to $120 \mu \mathrm{m}\left(1 / \mathrm{e}^{2}\right.$ value $)$. As predicted, the curve recorded with equal power has a larger recording slope than the curve recorded with equal intensity and saturates to a much lower diffraction efficiency (dashed curve). For the case of equal intensity in the reference and signal beam, the localized hologram growth can be written as [10]: 


$$
\sqrt{\eta^{\prime}}=\frac{A_{o}}{\sqrt{M}}\left(1-e^{\frac{2 t}{\tau_{11}}}\right)
$$

Where $M$ is defined as the ratio of the crystal length $\mathrm{L}$ and $\mathrm{L}$ ' which is defined as the $1 / \mathrm{e}^{2}$ lateral extension of the focused reference beam. $A_{o}$ is the saturation diffraction efficiency of a hologram with extension $L$ (a distributed volume hologram). $\tau_{w}$ is the recording time constant of a distributed volume hologram of extension L. From Eq. 3, the maximum diffraction efficiency of a single localized hologram is $\eta^{\prime}=\mathrm{A}_{\mathrm{o}}{ }^{2} \mathrm{M}$. If we assume that subsequent recording do not erase previous hologram. the final diffraction efficiency after $M$ exposure varies as $1 / M$. Localized hologram can thus be written with strong diffraction efficiency. This is achieved at the expense of a much longer recording time. The total recording time is equal to $\mathrm{M} \cdot \tau_{\mathrm{W}}$ which is a factor $\mathrm{M} / \mathrm{ln}(\mathrm{M})$ longer than when recorded using an exposure schedule using volume holograms.

We experimentally recorded 50 localized holograms with equal writing intensity in the reference and signal beam. Localized hologram are recorded in a $0.85 \mathrm{~mm}$ thick, transmission geometry, $\mathrm{LiNbO}_{3}$ crystal doped with $0.075 \mathrm{wt}_{0} \% \mathrm{Fe}_{2} \mathrm{O}_{3}$ and 0.015 wt. \% MnO. We use green recording wavelength at $532 \mathrm{~nm}$ with a reference beam focused by a cylindrical lens yielding a lateral extension of $30 \mu \mathrm{m}\left(1 / \mathrm{e}^{2}\right.$ value). The signal has a diameter of $3 \mathrm{~mm}$ at the crystal. Each hologram is recorded up to saturation for a recording time equal to two writing time constants. Center to center spacing of each hologram is $60 \mu \mathrm{m}$. The total spatial multiplexing is done over $3 \mathrm{~mm}$. Fig. 7 shows the diffraction efficiency of each recorded localized hologram immediately after each recording. An average value of $2 \%$ diffraction efficiency is achieved. Fig. 8 shows the final diffraction efficiency after the recording of 50 holograms. The average diffraction efficiency is about $110^{-3}$.

We can explain the loss of diffraction efficiency measured from fig. 7 to fig. 8 by three factors. The first factor is a drop caused by the erasure of the grating in $\mathrm{Fe}_{2} \mathrm{O}_{3}$ accounted by a factor 2.5 from experiments. The second factor is due to the finite erasure time constant of the grating in Manganese due to slow erasure by green light. Experimentally, this is accounted by a loss factor equal to 4 . A third factor is the erasure caused by the sensitizing wavelength to adjacent holograms. This factor varies with the center to center spacing of localized holograms. Figure 9 shows an example of the effect of the sensitizing beam on adjacent holograms. Although the adjacent holograms are located a distance equal to twice the spot size of the reference beam, the adjacent holograms suffer from erasure due to the sensitzing beam. In this experiment, the loss due to this effect is accounted by a factor approximately 2. Overall, the saturation diffraction efficiency of each localized holograms is decreased by a factor 20 and explain the values obtained experimentally. The $M$ number for this experiment is given by $\mathrm{M} \#=\mathrm{M} \cdot 10^{-32}=1.6$. This result has to be compared with the $\mathrm{M} \#$ obtained by distributed recording which for extraordinary polarization in the same crystal is $\sim 1$ [3]. The main problem to solve in order to obtain larger M\#\# using localized recording is related to the sensitizing light. Since the focused beam has a gaussian shape, the localized holograms are spaced by twice the waist of the focused beam in order to avoid erasure of adjacent holograms while recording the next hologram. Even though, with a center to center spacing of twice the waist, the erasure of adjacent holograms caused by the sensitizing beam still have strong effect.

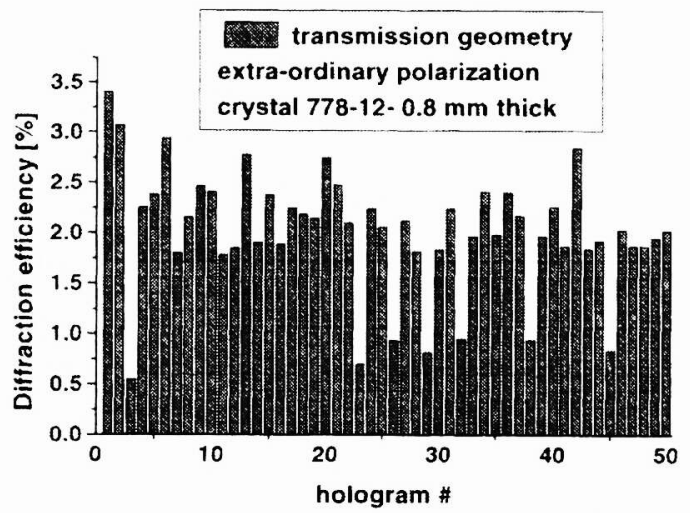

Figure 7: Diffraction efficiency of each localized hologram measured after each recording

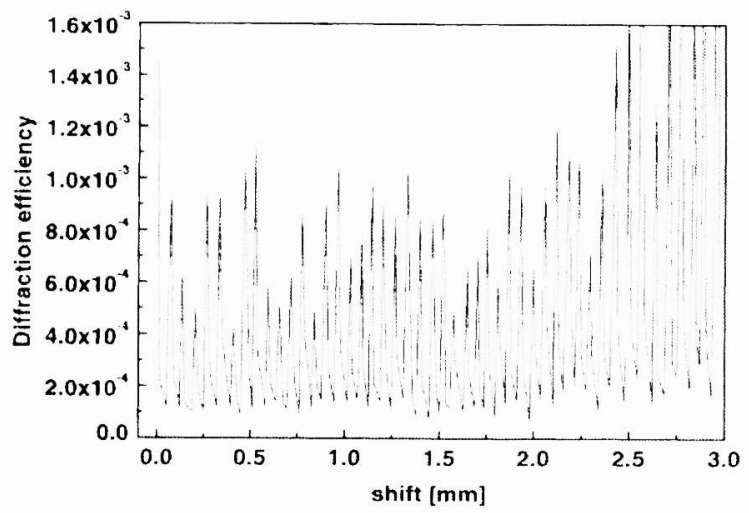

Figure 8: Diffraction efficiency after recording 50 holograms 


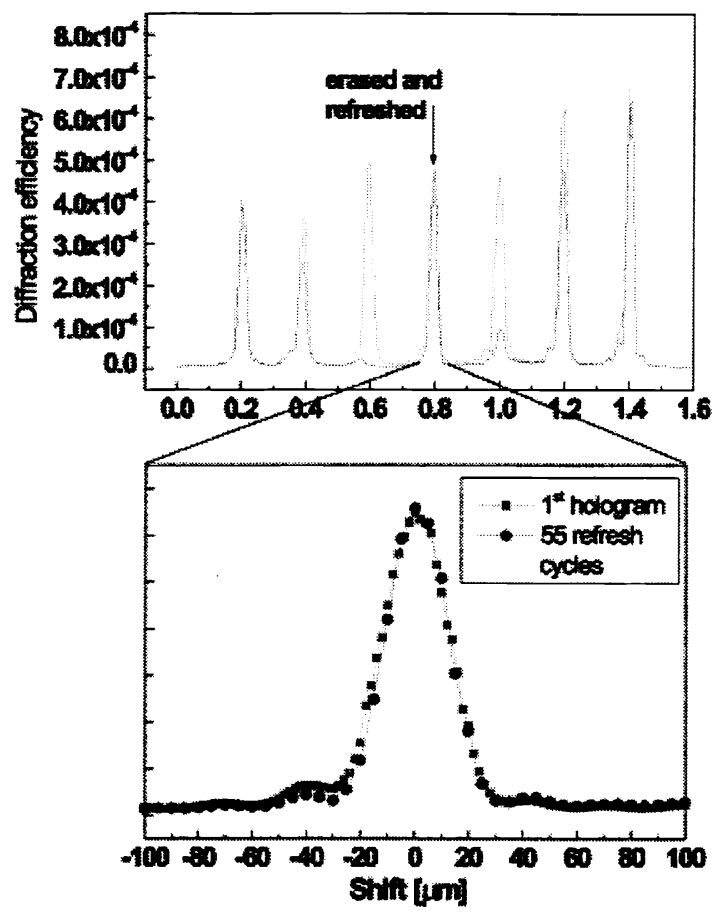

Figure 9: Erasure effect of the sensitizing beam on adjacent holograms

\section{REFERENCES}

[1] G.T Sincerbox, Selected papers on Holographic storage, SPIE Milestone Series, vol. MS 1995.

[2] K. Buse, A. Adibi, D. Psaltis, "Non-volatile holographic storage in doubly doped lithium niobate crystals" Nature. 393, 665, 1998.

[3] A. Adibi and K. Buse and D. Psaltis, "Multiplexing holograms in LiNbO3 : Fe : Mn crystals" Opt. Lett. 24, 652,1999.

[4] C. Moser, B. Schupp, D. Psaltis, "Localized holographic recording in doubly doped lithium niobate", Opt Lett. 25, 162164,2000

[5] H. Guenther and G. Wittmann and R.M. Macfarlane and R.R Neurgaonkar, "Intensity dependence and white-light gating of two-color photorefractive gratings in LiNbO3 “ Opt. Lett. 22, 1305, 1997.

[6] K. Buse and L. Holtmann and E. Kratzig, "Activation of $\mathrm{BaTiO}_{3}$ for infrared holographic recording" Opt. Commun. 85, 183, 1991.

[7] L. Hesselink and S. Orlov and A. Liu and A. Akella and D. Lande and R.R. Neurgaonkar, "Photorefractive materials for nonvolatile volume holographic data storage" Science. 282, 1089, 1998.

[8] D. A. Parthenopoulos and P. M. Rentzepis, “3-Dimensional optical storage” Science. 245, 843, 1989.

[9] M. M. Wang and S. C. Esener and F. B. McCormick and I. Okgšr and A. S. Dvornikov , P. M. Rentzepis” Experimental characterization of a two-photon memory", Opt. Lett. 22, 558, 1997.

[10] F.H. Mok and G. Burr and D. Psaltis, "System metric for holographic memory systems"

Opt. Lett. \{lbf 21,\} 896 (1996). 